\title{
Design and Implementation of the High Pressure Valve Automation
} Test System

\author{
Yiduo Wang ${ }^{1,}$, Feng Luo ${ }^{1, b}$ \\ ${ }^{1}$ Clean Energy Automotive Engineering Center, School of Automotive Studies, Tongji University, \\ Shanghai, 201804, China \\ aemail: wyd199001@163.com, bemail:luo_feng@tongji.edu.cn
}

Keywords: High Pressure Valve; Automation Test; OPC

\begin{abstract}
These days, there emerges an increasing stringent requirement for the pressure-resistant properties as well as gas tightness properties of high pressure valves in industrial productions. Traditional test procedures are implemented in forms of manual or semi-automatic manner, where several disadvantages exist, such as low test efficiency and insufficient test reliability. Therefore, this paper designed a valve automation test system to get rid of the problems mentioned above as well as to provide more automation functionalities. The test process and result information demonstrated the designed system offered much more convenience as well as a higher precision and reliability than traditional manners.
\end{abstract}

\section{Introduction}

The pressure resistance and air tightness test for valves act as the corner stone in guaranteeing safety production and promoting product quality. The application of these tests has already been expanded from the traditional automobile industry to the daily necessities industry, food processing and packaging industry, medical device industry, etc [1].

The traditional test methods consist of the bubble counting and soapy water smearing. Although these two methods are easy to implement, there still exist some disadvantages, such as the inaccuracy measurement of the leakage, low efficiency of the manual operation, poor versatility and so on. To improve the automation performance of the test system, an automated test system has been designed on the basis of SCADA software and PLC [2] [3]. However, due to the limitation of the automation function of this kind software as well as the complexity in parsing the communication protocol, this scheme is not regarded as the best choice.

In this paper, plenty of automation functions are implemented. To avoid the complexity of parsing communication protocol, OPC technology is utilized to simplifying programming [4] with a 187.5kps communication speed. Besides traditional automation functions, more features such as system simulation, hierarchy alarm system, results query and report printing are implemented with industrial automation controls, boosting the system safety coefficient and automation level.

\section{System Hardware Introduction}

The brief structure of this system can be illustrated in Fig. 1. The hardware of this system could be divided into three parts: a control subsystem, an execution subsystem and a monitoring subsystem. The control subsystem, in charge of monitoring and controlling the execution subsystem, mainly incorporates a PLC module, an IPC module and a cabinet panel. The execution subsystem, consisting of such sensors and actuators as pressure transducers, temperature transducers, electromagnetic valves, etc., receives instructions from the control subsystem and implements the automation test process. The monitoring subsystem is constituted by an IPC, a router and IP cameras, which reflects the bubbles in the water bottle during the seal test to provide references for the gas tightness of the valve. Specific details of PLC module and transducers are described below.

PLC Controller. This system adopts the SIEMENS CPU226 as the PLC controller. In terms of load capacity, the CPU226, integrated with 40 digital I/O pins (24 input pins and 16 output pins), is 
capable of connecting 7 extended modules with 248 digital I/O pins and 35 analog digital I/O pins [5]. As for the communication capability, this module supports PPI, Free-port, MPI, Profibus and OPC, which meets various communication occasions.

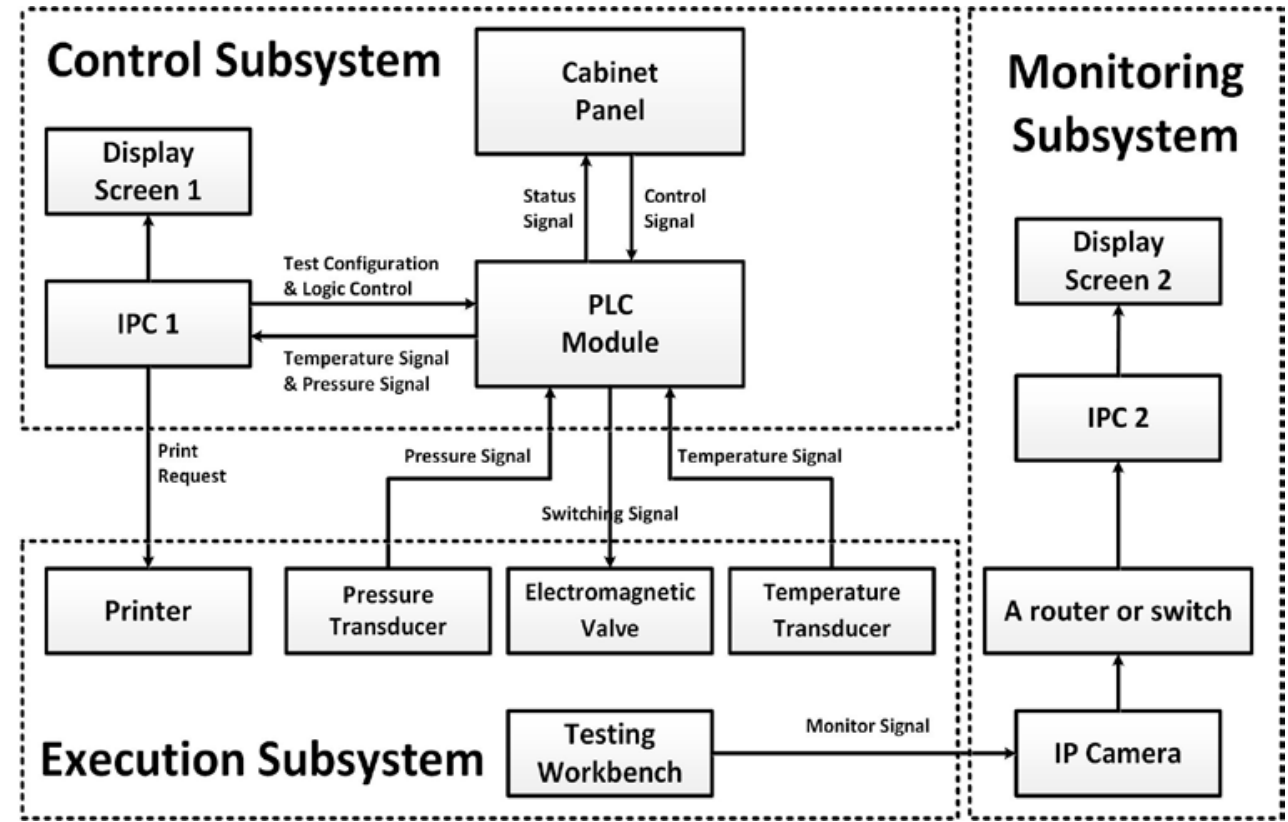

Fig. 1 System architecture

Extended Modules. As this system requires 32 digital output pins, 2 analog output pins and 10 analog input pins, 1 EM223 (32DI/32DO), 1 EM222 (8DO) and 3 EM235 (4AI/1AO) are employed to satisfy the I/O requirement. The digital module EM223, with $9 \mathrm{~W}$ power dissipation, supports a 24VDC/0.75A output and 24VDC/4mA input for each I/O pin, where the output ability is the same as EM222's [5]. The I/O pins of these modules are utilized to control and monitor the on-off state of such digital signal actuators as buttons, lights, magnetic valves and etc. In addition, the counterparts of analog module EM235 are 24VDC/60mA for DI and 24VDC/20mA for DO. Similarly, the I/O pins of this module act as controllers of the proportional decompression valves and monitors of the pressure and temperature transducers.

Transducers. This system employed a diffused silicon pressure transmitter with a $0-60 \mathrm{MPa}$ measuring range and $\pm 0.03 \% \mathrm{FS}$ measuring precision. The output signal of this sensor reaches 4-20mA, matching the current input mode of the EM235. As for the temperature sensor, the Pt100 with a $0-150{ }^{\circ} \mathrm{C}$ range and $\pm 0.2 \%$ FS precision is adopted to transmit a $4-20 \mathrm{~mA}$ signal to reflect the environment temperature.

\section{System Software Structure and Function Introduction}

This system is a high pressure valve testing system integrated with testing and monitoring modules, targeting the pressure and tightness test. Its software consists of a PLC actuator program and a PC client. Fig. 2 shows its software architecture.

PC Software. The PC client software is developed using Delphi language and SQL Server, which includes the design of user interface, database structure and testing result report template as well as the programming of system operation and valve test logic. The user interface serves the functionality of interacting with users, transferring their operations to the corresponding program, then abstracting actual test conditions from the run and test logic module and, finally, displaying them. As for the run and test logic module, it takes three main responsibilities. Firstly, it processes users' operations, such as automatic test, test result print and result query, and then demonstrates relevant information on the user interface. Secondly, it takes charge of system state transitions, such as running modes and alarm status. At last, the valve test process is also controlled by this program via the OPC server so that the system test information is transmitted to user interface.

OPC Communication. When it comes to the communication, the OPC controls, such as Iocomp, 
act as the OPC client to connect the PC Access (OPC server) and exchange user operations and test data between the PC and PLC.

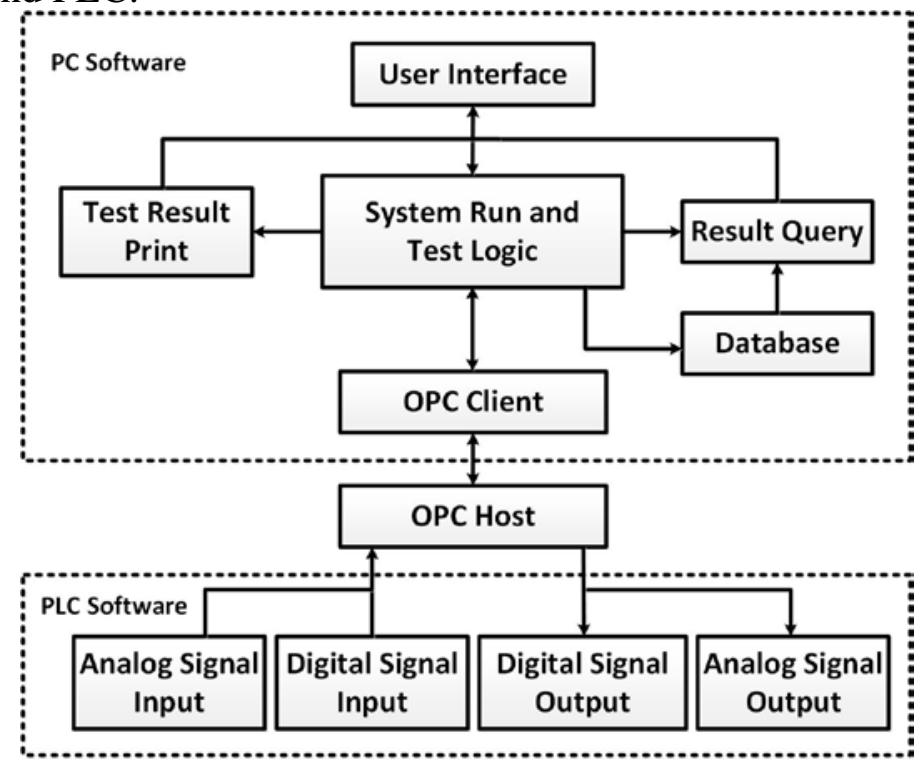

Fig. 2 System software architecture

PLC Software. The PLC software is developed using the ladder language in the Step7, which is comprised by the input and output of the analog and digital signals programs. Specifically, the temperature and pressure data is acquired by the analog signal input program while the button and switch input is obtained by the digital signal input one. In terms of the output program, the proportional decompression valve is controlled by the analog signal output program while the state of the magnetic valve depends on the digital signal output one.

\section{System Working Principles}

The operational procedure of this system is as follows. Step one, the operator sets such testing parameters as, station, pressure, duration, etc. Step two, the user starts automatic testing procedures operating in accordance with the testing parameters and logic provided by the PC client. In this step, the booster pump, automatically and roughly, pressurizes the system while the decompression valve regulates the station pressure precisely. Step three, once the station pressure reaches the testing pressure set previously, the pressure holding procedure, whose time expenditure conforms to the testing duration, starts immediately. Accompanying with step three, the pressure-time and temperature-time curve is depicted and stored in the database for historical data queries. Step four, if the pressure holding process finishes, the system decompression procedure starts and the testing report printing request is transmitted to the printer. Step five, the whole testing process completes when the pressure relief phase ends and all the actuators recover to their initial states.

\section{The Realization for the Main Functions of the System}

System State Information Display and Control. The system state information consists of three kinds of records, the pressure value, temperature value and the state of the electromagnetic valve. Among these signals, the analog quantity is firstly converted into digital one, which is then uploaded with other digital quantities to the PC software through the OPC host. After that, these state records would be illustrated in the PC client interface. On the basis of the information mentioned above, the valve testing logic in the software serves its own functions, such as conducting corresponding system state transitions or sending relevant control signals to the PLC module. Once the control signals are received from the PC software, the PLC would carry out the corresponding executive program in response to the workflow action.

Testing System Alarm Function. Either the pressure value in the air compression station, cylinder bundles, valve chamber and so forth is insecure, or the pressurize duration is too long, or 
something unstable and abnormal happens, the alarm mechanism should be established so that the system can give feedbacks of abnormalities to the user. To detect these alarms, the alarm parameters need to be assigned. Upon the testing system runs, the PC client builds a connection with the database which saves the alarm parameter configuration, and compares thresholds in database with the real-time pressure, temperature, pressurize duration, maintain pressure drop, etc. then alarms when either of the actual running indexes exceeds the predefined arguments.

Air Tightness Test Video Monitoring. When the system is adopted for conducting the valve chamber air tightness test, the possible gas leakage would lead to the water bottle downstream the testing valve, which is under the supervision of the integrated monitoring subsystem. Having the IP camera being connected with the IPC via a router, the real-time supervisory signals are uploaded to as well as saved in the computer for viewing online.

Historical Data Query and Report Print. Once the testing procedure finishes, the corresponding results are preserved in the form of data record in the database. When the user queries information in the historical inquiry window, the connection between the PC software and the database is established and then the related testing results information would be searched and demonstrated in the query display panel. Meanwhile, the PC client would transfer those data to a report template and create the query results summary report when a print request received.

\section{The Implementation of Test System and the Results Analysis}

The real test system is shown in Fig. 3.

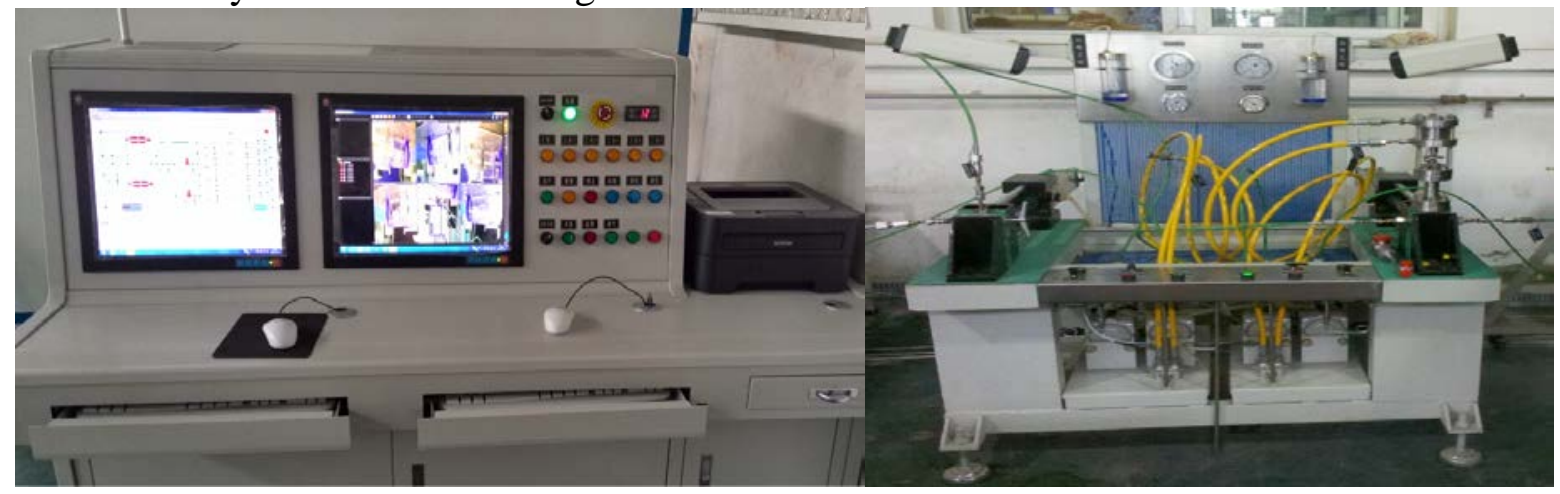

Fig. 3 Real Control and Monitoring System and Real Execution System

The real PC client software can be illustrated as Fig. 4 which shows the system and workstation running status respectively.

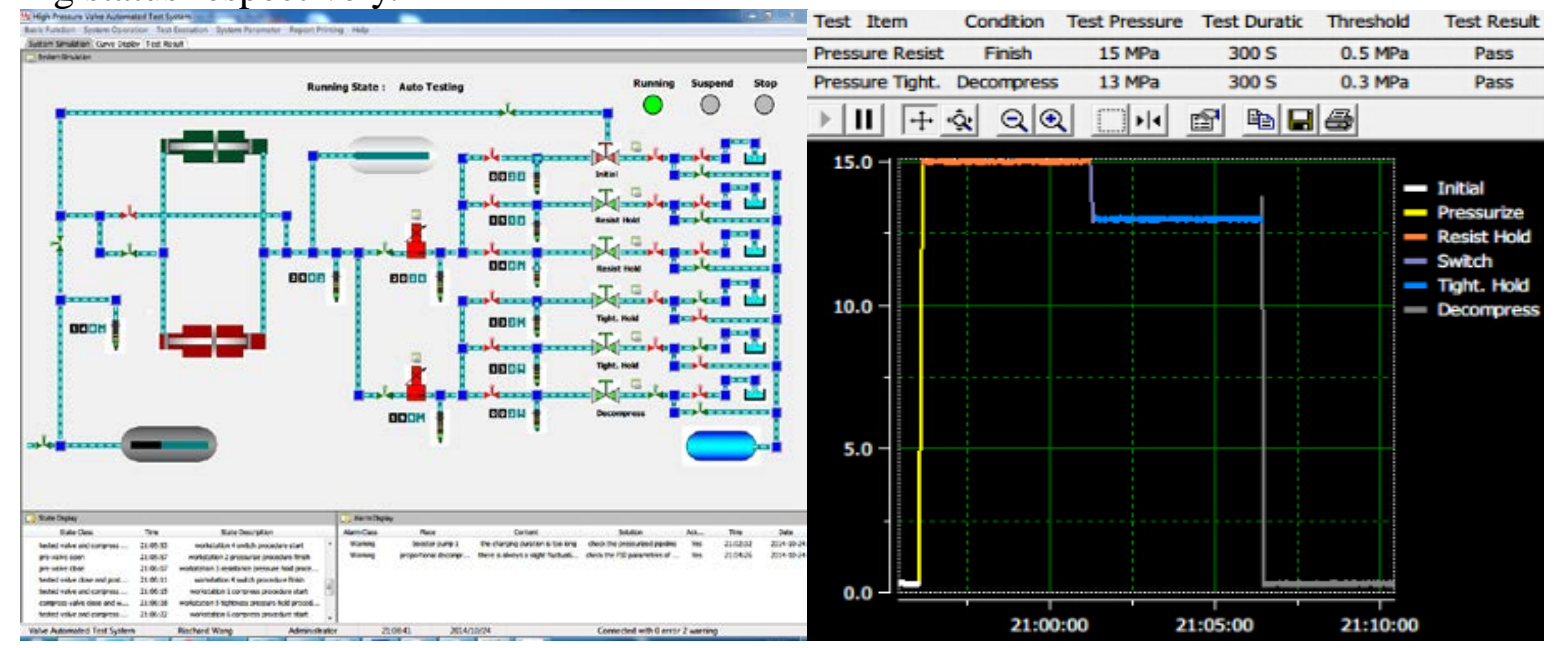

Fig. 4 Left: System running status. Right: Workstation running status

Fig. 4 (Right) demonstrates a pressure curve generated in the automatic test mode which reflects the test parameters, pressure information as well as the result details of the whole test process.

The overall testing procedure is divided into 5 stages:

Pressurized Stage. In this stage, with the outlet valve downstream the tested valve closed, and 
the tested valve plug as well as the inlet valve upstream opened, the high pressure test gas is guided into the intake pipe and therefore the pressure in the valve chamber becomes increasingly higher.

Pressure-Resistance Pressure Holding Stage. If the growing pressure in the valve box meets the testing requirement, the inlet valve closes, which means the start of the pressure-resistance pressure holding stage. The purpose of this phase is to test the pressure resistance property of the valve. If any air leakage problem appears in this phase, the stress in the cavity would experience a dramatic loss during the pressure holding stage. If the chamber stress decline is within the acceptable range of leakage rate during this stage, the tested valve is proved eligible in this test. In case the pressure drop meets or exceeds the inferior limit of the valve testing criteria, it can be proved that the tested valve is of disqualification in the pressure resistance property.

Switch Stage. Upon the pressure-resistance stage ends, the testing procedure enters the switch stage for the preparation of the air tightness pressure holding phase. In this stage, the tested valve element and the inlet valve are closed while the outlet valve is open, which keeps the pressure stay in the upstream chamber of the valve and releases the gas behind the valve plug to the atmosphere.

Air-Tightness Pressure Holding Stage. The testing procedure enters this phase once the stress after the valve element descends to the atmospheric pressure while the counterpart in front of the plug remains the same. As this stage objective is to guarantee that the pressure tightness is properly served, the valve plug and the inlet as well as the outlet valve are switched into closed state while the valve ahead of the water bottle opens so that any tiny gas leakage can be easily observed. On this occasion, the pressure in the chamber would witness a consistent and slow decrease of itself if there was a loophole in the tested valve element. In terms of the test result, this stage approximately shares the same eligibility criteria with the pressure-resistance pressure holding phase except that the test pressure and qualified threshold here is smaller than the counterparts.

Decompression Stage. Eventually, the main test processes finish and all valves on the workstation open to release the high pressure gas to the atmosphere. Once the pressure decreases to the safe area, all valves of the station switch to closed state and this test procedure completes.

\section{Conclusion}

This paper designs an automation test system for high pressure valves to eliminate the inefficiency and invalidity existing in the traditional ones. Apart from those, more automation functions are implemented to afford more convenience to the system usage. In order to display the system state in real-time and to promote the safety performance, the system simulation function is implemented and a hierarchy alarm system is also designed to locate the precise alarming source. The curve display function provides records during the test process in details for data analysis to boost the test effectiveness while the test result query interface realizes the backtracking of the test information and files. According to the test process and result analysis, this system achieves highly automatic test operations and the test results also truly reflect the mechanical properties of valves.

\section{References}

[1] Lu Peiwen. Test and inspection of valves [M]. Beijing: Standards Press of China, 2010.

[2] Yang Yang, Cheng Aihua, Chen Zhenyi. Design of Control System for the Valves in Multi-test Bench of Screw Pump Based on MCGS and PLC [A]. Development \& Innovation of Machinery \& Electrical Products, 2012.

[3] Yu Meng, Zou Zhiyun, Zhao Dandan. Design of Automatic Valve Test Control System Based on PLC and Touch Screen [B]. Automation in Petro-Chemical Industry, 2013.

[4] Chai Kai, Hou Ligang, Jiang Junyin. Research on the application of the OPC technology to industrial process control [A]. Industrial Instrumentation \& Automation, 2005.

[5] SIEMENS SIMATIC S7-200 programmable controller user manual. Siemens AG, 2008. 